\title{
OPENINGS IN QUITE FORMAL SCIENTIFIC TEXTS
}

\author{
MICHAEL P. JORDAN \\ QUEEN'S UNIVERSITY AT KINGSTON
}

\section{BACKGROUND, AIMS AND BASIS}

Earlier work describing openings in short, informal scientific texts in New Scientist (Jordan 1991a) explains how the writers usually concentrate on aspects of problems, solutions and evaluations in the titles and opening remarks. A few of those texts indicated broader aspects in their openings, and this has led to the present study of much larger, more formal texts. The aim is to describe and explain, within established linguistic systems and within known principles of scientific enquiry, the major methods used by skilled scientific writers and editors to introduce the subject, convey the major scientific principle(s) involved, and interest prospective readers.

Major articles from Scientific American have been chosen as the basis for this study. This publication has a well-earned reputation not only for clear, organized scientific prose, but also for meaningful and interesting accounts of current scientific issues and developments. Their major articles are highlighted with photographs in a list of contents, and contain brief one- or two-sentence abstracts as well as noteworthy introductions. This study concentrates on the title, the abstract, and the opening few paragraphs of the introduction in a search for systematic linguistic and rhetorical devices which could be regarded as typical of exemplary openings in quite formal scientific texts.

The term "quite formal" has been chosen with care. The openings analyzed for this study are certainly more formal than the brief minireports analyzed in the earlier study. But they also contain many elements of style and rhetoric which are not usually found in very formal scientific papers. Openings in very formal scientific texts could be the 
basis for a further study, which would concentrate much more on specific scientific methodologies and verification principles than we find in articles for Scientific American.

For this study, the longer articles published during 1990 have been selected, but the series of articles on energy in the September issue and on possible causes of mass extinction in the October issue have been excluded as these exhibit features special to series rather than standalone articles. Throughout this paper, the origins of examples cited are indicated first by the first three letters of the month of the issue, then the page number, and then by line(s) in the title (t), the abstract (a) and the introduction (by paragraph). Thus Nov $44 \mathrm{t} 2$ indicates the second line of the title on Page 44 of the November 1990 issue, Aug 34 al-2 indicates the first two lines of the abstract on Page 34 of the August 1990 issue, and Apr 66 2/3-6 indicates the third to sixth lines in the second paragraph of the introduction on Page 66 of the April 1990 issue. My underlining in the examples cited highlights key connecting words.

The openings in the articles studied are described here within three established meta-systems of language understanding: the "logical connections" of problem-solution, purpose-means, enablement, causeeffect and basis-assessment; narrative and such organizational pairs as comparative denial, concession, denial of the expected, known-new, and hypothetical-real; and other opening strategies and rhetorical devices. The final section of the paper summarizes the findings and gives guidelines and advice for teachers of technical writing and writers of such articles.

\section{LOGICAL CONNECTIONS}

The "logical" connections used here are discussed in more detail elsewhere (e.g. Jordan 1986, 1991b), with problem-solution patterns in Winter 1976, Hoey 1983 and Jordan 1984, for example, enablement in Mann and Thompson 1986 and cause-effect signalling in Jordan 1989. In summary, problem-solution deals with difficulties and how they are overcome, purpose-means deals with goals and their achievement, causeeffect deals with scientific interaction of events, basis-assessment deals 
with thoughtful conclusions and their basis or evidence, and enablement is the action making something possible or permissible.

As a bridge between this present study and the earlier work on informal texts (Jordan 1991a), problems and solution concepts are considered first. As the texts studied here are in fact the more detailed (and thus more formal) discussions summarized in the earlier work on informal scientific texts, it should come as no surprise that again problems and solutions are significant factors in many of the articles. Under a title of Third World Ballistic Missiles (Aug $34 \mathrm{t}$ ), we find a clear problemsolution pattern:

(1) The spread of strategic weapons to unstable regions greatly increases the likelihood that they will be used. We cannot reverse the process and so must learn to manage its consequences (Aug 34 a)

This abstract recognizes a problem, rejects one solution (reverse the process), and then announces the proposed solution. The introduction explains the growth of arms capabilities in Iraq and Israel as the basis for a clear statement of the problem:

(2) Ballistic missiles and other means of long-range destruction, traditionally limited to a handful of industrialized nations, are fast becoming a fixture in many regional conflicts. The Third World military buildup is perhaps even more worrisome than its First World prototype, for it is more likely to find expression in war. (Aug 34 2/4-13)

As recognition of a problem is an assessment, and in science assessments are given basis, the article then provides several reasons why this should be so (Aug 34 3/1-2) before discussing the recommended solution to the problem.

Interactive problems, where solutions cause a problem to someone or something else, are also common features of the informal and these quite formal texts. After a clearly problem-signalling title Chestnut Blight (Jul $106 \mathrm{t}$ ), the abstract explains the cause of the problem, and how a 
new parasite could be the solution to the problem by controlling the cause:

(3) A fungus that has ravaged the American chestnut is itself coming under biological attack. Plant pathologists hope the new parasite will eventually control the old one (Jul 106 a)

Even from these brief extracts, it should already be clear that the openings contain complex combinations of connections. Recognition of the problem in Example 1 is an assessment for which basis is given in the article, and cause-effect elements can be seen in Example 3. For purposes of clarity, the analysis concentrates on the main theme being discussed at the time, with other complexities being noted in passing.

We must now recognize a special type of problem: that concerned with the need to know something. Such "intellectual" problems are often clearly signalled as such; and their solutions, of course, are greater understandings, which are the incentives for all scientific enquiry. The following extract poses an intellectual problem by challenging existing knowledge:

\section{The Solar-Neutrino Problem}

Painstaking observations show that the sun emits fewer of these elusive particles than theories predict. This deficit challenges current understanding of physics and of the process by which the sun shines (May $54 \mathrm{t}$; a)

The lack of knowledge is clearly signalled as a "problem". After a brief description of neutrinos and the need to study them, the article expresses this intellectual problem as a flaw in our knowledge and then explains the importance of solving this problem:

(5) The message that the solar neurtrinos can carry is puzzling and provocative: some flaw seems to exist either in the current models of the sun or in our present understanding of the laws of physics. Solving this mystery may reveal new clues as to how the sun shines, 
how stars evolve and whether the forces of nature are fundamentally related. (May 2/8-17)

Principles of purpose and means (also called "instrument") are very closely related to those of problem-solution (Jordan 1985a). Our purpose is often to solve the problem, and the means of achieving this purpose is the solution to the problem. The closeness of the two pairs of relations is shown by:

\section{Gene Therapy}

Treatment of disease by introducing healthy genes into the body is becoming feasible. But the therapy will not reach its full potential until the genes can be coaxed to work throughout life (Nov $68 \mathrm{t}$;a)

Disease is the problem and gene therapy is the solution. The means clause (by introducing...the body) rank-shifted into the subject of the first sentence indicates the means of solving the problem. The But introduces a problem with this solution, which will have to be solved.

Although by...ing and by means of are typical signals of the purposemeans relation, it is often communicated by signalling the purpose (e.g. To treat disease, healthy genes are being introduced into the body.). Two such clauses are seen in:

\section{Adoptive Immunotherapy for Cancer}

Also called cell-transfer, it is one of a new class of approaches being developed to strengthen the innate ability of the immune system to fight cancer (May $62 \mathrm{a} ; \mathrm{t}$ )

The relation of enablement is a sort of solution to the need to do something, and is the means by which freedom is conveyed to allow achievement of a purpose. This complex concept and its connection with purpose is shown by:

(8) The abundance of data will enable physicists to probe their present understanding of physical law as never before (Jul 54 t2-3) 
A biochemical mechanism called the water-oxidizing clock enables plants and some bacteria to exploit solar energy to split water molecules into oxygen gas, protons and electrons (Feb $50 \mathrm{t} ; \mathrm{a}$ )

This last example contains signals of means (How), and purpose (to) as well as enablement. Partial enablement (help), purpose (to mount) and means (by triggering) combine with problem (micro-organism and invader) and solution (defense and the multiplication of only those cells that attack) in the following example:

\section{Interleukin-2}

The first hormone of the immune system to be recognized, it helps the body to mount a defense against microorganisms by triggering the multiplication of only those cells that attack an invader (Mar $50 \mathrm{t} ; \mathrm{a})$

At first sight, the relation of cause-effect appears almost separate from the complexities and inter-connectedness we are beginning to recognize:

The Variable Sun

Its steady warmth and brightness are illusory; the sun's output of radiation and particles varies. Systematic observations are beginning to unveil the causes of these changes and their effects on the earth (Feb $34 \mathrm{t} ; \mathrm{a})$

However a closer look at this example reveals a change in the understanding (assessment) and basis (Systematic observations) which will enable scientists to solve their intellectual needs to understand both the causes and the effects of the variations. Much greater connectedness between cause-effect and problem-solution is often needed to explain the thrust and background of the work described in the article: 
Government policies that encourage exploitation - in particular excessive logging and clearing for ranches and farms - are largely to blame for the accelerating destruction of tropical forests (Apr 36 $\mathrm{t} ; \mathrm{a})$

Here we have a problem in the title amplified at the end of the abstract. But we are concentrating on the cause, and thus the problem is seen as the effect. The thrust of this article is made clear in the introduction:

(13) Tropical forests are disappearing at a rate of tens of thousands of square miles per year....Among the agents of the devastation are the inefficient commercial logging operations and the conversion of forested areas to cattle ranching and agriculture....both the logging and the conversion are the result of government policies. Many of these policies are driven by the severe economic pressures afflicting debt-burdened under-developed countries. Those pressures in turn are exacerbated by certain practices of developed countries... (Apr $361 / 1-3,2 / 1-5$ and $8-16$ )

Thus we have two causes for the problem, a single cause for these two causes, and an initial cause; this all provides basis for the writer to make the assessment that Hence the causes as well as the effects of tropical-forest degradation should elicit world-side concern $(2 / 18-20)$.

We have already seen examples of assessment-basis, which includes the conclusion or decision together with the evidence, data, grounds or reasoning used to arrive at the assessment. Evidence in the form of manuscripts is seen as sole and complete basis for the account of a city's life in:

(14) Among the finds are 700 birch-bark manuscripts that give an intimate view of the city's life (Feb 84 12-3)

More typically evidence provides only clues to a greater understanding: 
(15) Atomic clusters can offer clues to freezing and melting (Aug 68 a1) and mechanisms in one branch of science may yield clues in another:

(16) A variety of animals freeze solid during the winter months and thaw in the spring. This natural ability to survive freezing may yield clues to the cryopreservation of human issue (Dec 92 a)

In particular, research on animals in the wild is an established method of providing insights into human behaviour:

$$
\text { Stress in the Wild }
$$

Studies of free-ranging baboons in an African reserve are helping to explain why human beings can differ in their vulnerability to stressrelated diseases (Jan 116 ta)

Assessment and basis lie at the heart of scientific analysis, debate, controversy - and of course all publications that explain these. Often new evidence supports the established theory:

(18) The theory that all humans are descended from a recent African ancestor was promoted by geneticists who study living populations. The fossil record provides independent support for this model (Dec 98 a)

But newly-researched or newly-discovered facts can form the basis for modification or rejection of established theory:

\section{Unconscious Mental Thinking}

Rigorous, quantitative studies of psychotherapy are challenging widely held views of how the unconscious mind works and how patients in therapy make progress (Mar $103 \mathrm{t} ; \mathrm{a}$ )

The challenge to traditional thinking is amplified in the introduction: 
(20) It is generally assumed that human beings cannot carry out unconsciously the same kinds of intellectual activities they perform consciously, such as making plans and assessing risks. Yet our studies of patients in psychotherapy indicate that, in fact, people can unconsciously think, anticipate consequences and make and carry out decisions and plans. (Mar $1032 / 10$ )

A challenge is one thing, but occasionally new evidence can totally destroy a premise and a whole line of reasoning. In the following, the discovery of a factory blows away a complex reasoned myth:

\section{A Roman Factory}

Most historians hold that the Romans failed to develop the technology of mechanized production because slave labour was so cheap. The ruins at Barbegal in France tell another story (Nov 106 $\mathrm{t} ; \mathrm{a})$

And a new understanding can actually blur rather than clarify the larger framework of understanding:

\section{The Early History of Indo-European Languages}

The common ancestor of these languages has been traced to Asia rather than Europe, the authors say. The once-clear distinction between the family's Eastern and Western branches is now blurred (Mar 110 t;a)

\section{OTHER LINGUISTIC SYSTEMS}

The key to understanding differences and changes in knowledge lies with concepts of comparative denial pioneered by Winter (1974, 1982) and Hoey (1983). In basic form the relation between two statements is connected by the idea that what is true of $X$ is not true of $Y$, where $X$ and $Y$ can be different things, times, places, or circumstances. For this application, what is true at time $X$ is seen to be not true of time $Y$ (a later time) as our understanding improves over the years. A clear twopart example of this type is: 
(23) When Arthur Ashkin and his colleagues at Bell Laboratories first noticed the photorefractive effect some 25 years ago, they considered the phenomenon a curiosity at best and a complete nuisance at worst. Today photorefractive materials are being shaped into components for a new generation of computers that exploit light instead of electricity. (Oct 62 1/1-10)

With more than two items of change as a function of time, we have narrative, a time-oriented discussion of events. But although narrative is a common means of introducing the subject, it is a special kind of narrative in which progressively more refined theories are developed, overturned and replaced as scientific knowledge advances. Here is such a narrative structure:

(24) Throughout history human beings have sought to understand the meaning of dreams. The ancient Egyptians believed dreams possessed ocular power...Other cultures have interpreted dreams as inspirational, curative, or alternative reality.

During the past century scientists have offered psychological and neuroscientific explanations for dreams. In 1900 ...Freud proposed that dreams were the "royal road" to the unconscious...More recently, in contrast, dreams have been characterized as meaningless...

Based on recent findings in my own and other neuroscientific laboratories, I propose that dreams are indeed meaningful. (Nov $861 / 1-5$ and $8-10 ; 2 / 1-4,6-8$ and $10-13 ; 3 / 1-4)$

The use of in contrast in this example is a clue to a number of relations commonly used in the introductions of these articles. There is a clear contrast here between the different views of dreams, but in other examples "contrast" words (but, yet, however, nevertheless, etc.) are used to mediate between types of information other than simple contrast. Use of such connectors for these and other functions is discussed in Jordan 1985b. Closely connected to contrast is the connection involving what we do know and what we do not know: 
(25) When the mercury dips below zero degrees Celsius, we retreat to our warm houses...Birds have flown south and many terrestrial animals hibernate in dens or on lake bottoms. But what happens to ectothermic, or cold-blooded, animals - frogs and turtles, beetles and spiders - that cannot find a relatively warm haven? (Dec 92 $1 / 1-3$ and $7-14)$

Connection between previously "known" (or at least commonly accepted) information and new information is clearly structured in the following example with the sentence dominated by But indicating the transition between the two types of material:

(26) Almost every solid has a melting point, and almost every liquid has a freezing point. These two points are the same, just viewed from different perspectives...Hardly anything could appear simpler.

But appearances can be deceiving. Small clusters of atoms or molecules are offering new clues about melting and freezing points. (Aug $1 / 1-5$ and $11 ; 2 / 1-4$ )

A further relation often signalled this way is concession, which involves the denial and correction of an expected conclusion:

(27) Charleston, South Carolina, is a very long way from earthquake country. The rim of the Pacific, the Mediterranean and central Asia experience most of the world's earthquakes, both spectacular and small. Yet in 1886 a quake several times larger than the event that struck San Francisco in October of last year severely damaged much of Charleston. (Mar 68 1/1-10)

Given the location of Charleston, we would not expect an earthquake to occur there, but it did and it was a severe one. The denial of the conclusion we would expect to reach is signalled by Yet.

The concessive relation (involving the surprise when the expected conclusion is not achieved) is signalled very clearly indeed in the following not just by however, but also by To his surprise: 
(28) The year was 1936 . Hans Selye, a young physicist just starting off in research at McGill University in Montreal, had a major problem. He had been injecting rats daily with a chemical extract to determine the extract's effects and had identified consistent changes in the animals... To his surprise, however, the rats in the control group, which had been injected with saline solution alone, showed identical changes. (Jan 1161/1-8 and 11-14)

Overt denial of an expected statement (signalled by not) followed by what is correct is a closely-related method of starting the introduction. Two such structures occur in the following:

(29) The AIDS-causing human immunodeficiency virus (HIV) does not by itself produce most of the illness and death associated with the acquired immunodeficiency syndrome (AIDS). HIV can damage organs directly, but by progressively crippling the body's defenses, the virus also sets the stage for the development of opportunistic infections...Antiviral drugs that are effective against HIV, such as zidovudine (AZT), do not eradicate HIV completely, but they do slow the HIV-related decline in immunity. (Aug $501 / 1 \overline{0,2} / 1-5$ )

The "But-syndrome" is seen to operate when mediating between elements of the problem-solution structure:

(30) If nuclear war breaks out in the coming decade or two, it will probably be by accident. The threat of a cold-blooded, calculated first strike is vanishing but beneath the calm surface of constructive diplomacy among the traditional nuclear rivals lurks the danger of unpremeditated use of nuclear weapons. (Dec 53 1/1-9)

Another structure used for openings is "hypothetical-real" (Winter 1974), in which first a statement is made about what was thought to be true followed by what is true; this is related to what Grimes (1975) calls "collatoral inversion". Example 20 is a clear instance of this structure, with It is generally assumed that signalling the hypothetically and Yet indicating the transition from hypothetical to real. Actually a large group of relations is possible with this concept, often with But introducing an overt denial of the hypothetical statement before the 
"correction" of what is true. In the following example, a common misconception (signalled as such) that the bats' use of sound is crude is followed by (and signalled by But) the denial of that crudity and then (after the colon) the "correction" of what is true. The structure here parallels the then-now contrast in knowledge signalled by It used to be and since.

(31) It used to be a common misconception that bats' use of sound to navigate and locate prey is a crude system, the acoustic equivalent of feeling one's way in the dark with a cane. But biosonar has since been shown to be anything but crude: an echolocating bat can pursue and capture a fleeing moth with a facility and success rate that would be the envy of any military aerospace engineer. (Jan 60 1/1-11)

Here is a brief example of the simple hypothetical-real pair with implicit denial:

(32) To someone lying on the beach or taking a daytime stroll, the blazing sun seems constant and unchanging. Actually, the sun is a variable star. (Feb 34 1/1-5)

Larger elements of text are often connected in this way:

(33) Conventional economic theory is built on the assumption of diminishing returns...According to conventional theory the equilibrium marks the "best" outcome possible under the circumstances...

Such an agreeable picture often does violence to reality. In many parts of the economy, stabilizing forces appear not to operate. (Feb $921 / 1-3$ and $14-18 ; 2 / 1-4)$ 


\section{OTHER OPENINGS}

In the articles studied, the major logical connections in the body of the articles themselves are usually communicated in the abstract and occasionally also in the introduction. In contrast, the other linguistic patterns described in this paper occur mainly in the introductions and less frequently in the abstracts. In many cases, both of these patterns may be supported by initial introductory items, which cannot conveniently be classified into either of the two major linguistic groupings. These are discussed here.

The most significant of these other methods of introducing the article is by recalling a noteworthy event, and then explaining its significance to the purpose of the article. Here is a clear example:

(34) In 1872 railroad magnate Leland Stanford wagered $\$ 25,000$ that a galloping horse, at some point in its stride, lifts all four hooves off the ground. (Dec 76 1/1-5)

The article then explains how work enabled a horse flying through the air to be captured on film (a photo is included). This in turn provides an introduction to current methods of photography, by which we can now witness the motions of molecules as one substance changes to another (Dec 76 a3). The author has started with an interesting event and progressed to the current interesting situation, which has been placed in some perspective by the earlier event.

The above example is intended to attract interest. When, however, the event is a key one initiating an important line of inquiry, it becomes essential information to the substance of the article:

(35) In the early evening of November 21, 1974, powerful bombs ripped through two pubs in the industrial city of Birmingham, England, leaving 21 dead and 162 injured. The government immediately blamed the Irish Republican Army for the attacks and mounted a massive search for the perpetrators. (May 46 1/1-9) 
The article then explains how six men were convicted based on findings of forensic science, which were later found to be unreliable. This in turn raises troubling issues about the application of forensic technology to criminal investigations (May $463 / 1-4$ ), which are explored in the article under the thesis that Forensic methods must be screened with greater care if justice is to be served (May 46 a2-3).

Between the colourful event and the key objective event, we can find events recalled by the writer(s) which were part of their work described in the article -

(36) In January of 1981 we set sail from Woods Hole, Mass., heading to an area just southwest of Bermuda [to test oceanographic tomographic instruments] (Oct 94 1/1-3) -

or as a personal experience which spurred research discussed in the article:

(37) In 1968, when I was a surgical resident at a Boston hospital, I admitted a 63-year-old man complaining of abdominal pain characteristic of a gallstone attack. I then participated in the surgery to remove his gallbladder. It was a routine case except for one extraordinary feature. (May $621 / 1-8$ )

The account then explains how the patient, twelve years earlier, had terminal cancer, and that the cancer had since spontaneously disappeared, presumably through some immune mechanism. Such evidence, the writer says, has spurred many investigators to seek immunotherapies for cancer (May 62 3/9-11)

The hypothetical or "re-enacted" event or account is purely colourful, and is much more prevalent in general articles for less discriminating readers:

(38) A distant, muffled "whoomp" resounds, and a trail of yellow-orange sparks tumbles into the night sky, culminating in a circular burst of brilliant blue and green streaks. Another explosion shoots out a ragged arc of red streamers, followed by a shower of white and 
gold sparks. A third firework produces a staccato barrage of bright flashes of white light and thunderous noise. (Jul $961 / 1-11$ )

One group of openings provides introductory material which is not at all essential to the article, but which provides background, usually known, information - perhaps to allow readers to have a more equal starting point. In the following example, the main point of the article involves denial of the expected and its correction; this is made clear both in the abstract and in the second paragraph. But in the first paragraph we find some general information of little use to the main argument:

\section{Progress in Gallium Arsenide Semiconductors}

The compound is not a candidate to supplant silicon. Nevertheless, its speed and optical capabilities have spawned fast-growing applications in computing and communications.

Today's global age of electronics is built on a miniature foundation of microscopic circuits engraved on silicon chips. The current success and continuing promise of silicon in consumer, commercial, industrial and military electronic systems has prompted those who work with the material to offer a tongue-in-cheek criticism of another promising semiconductor, gallium arsenide. "Gallium arsenide," they say, "is the technology of the future, always has been, always will be."

After almost 30 years as the technology of the future, gallium arsenide has begun to make a place for itself, not by supplanting silicon but by complementing it in new applications. (Feb 68 $t ; a ; 1 ; 2 / \overline{1-5})$

It is the two by...ing means clauses, with denial of the expected and correction that develop the main purpose of the article as expressed in the abstract. The first paragraph serves little purpose.

This is not simply a unique aberration. In an article dealing with the need for vampire bats to feed on blood at least every two days, and 
discussing the solution adopted of sharing through regurgitation, we find the introductory paragraph:

(40) At night - long after most visual predators have stopped prowling - vampire bats emerge from their roosts and take to the wing, flying low across the landscape in search of warm-blooded prey. Within an hour or two, having found appropriate victims and fed on their blood, the bats return to the roost to sleep, feed their young and interact with nestmates. (Feb 76 1)

As the next paragraph is full of relational signals (Until recently, little was known, thought to be, however, and not known), the first paragraph is little more than a filler providing information readers already know. Much can be said for the use of quotations (as in Example 39) unless they bear directly and clearly on the main thrust of the article.

Descriptions of things as opposed to events could also lead to introductions that do little more than delay the main thrust of the article. But that is not always the case. In a detailed introduction of flight patterns of many insects (Nov 114 1/1-15), the description at first appears merely as a colourful flurry, but it does help readers to understand the extent of the manoeuvrability of insects before we finally get to the main point dealing with the means by which they do these things and the improving scientific knowledge of the subject. Thus known information may be acceptable as an introduction as long as it is made relevant to the main idea(s) of the article. We can see this in:

(41) To distribute their seeds far and wide, plants often enlist the help of animals. Some produce seeds that temporarily attach themselves to the hairs of mammals or the feathers of birds. Commercial orchard trees and some other plants have seeds that fruit-eating animals infest and that germinate when excreted or regurgitated. Vertebrates are not the only animals that disperse seeds, however - ants, too, are important.

Biologists are just beginning to recognize the specialized mechanisms that make ants a major force in the spread of plants around the world. (Aug 76 1;2/1-4) 
With the surprise of the compatibility being signalled by however, this introduction includes ants as important elements in the seed distribution process - and then indicates that their means of doing this will be the theme of the article.

\section{FINAL COMMENTS}

With problem-solution concepts widened to include interactive problems and intellectual "need-to-know" problems, the principles explained for openings in informal texts are also seen to apply to some quite formal texts. But we need to use the whole known system of logical connection in language to account for many more. Purpose-means, enablement, cause-effect and basis-assessment are all seen as important elements in some openings - and many instances were noted which involved very complex combinations of these methods of connection. Where logical connections were found, they were usually apparent in the abstract and usually also later in the text, but not necessarily in the first paragraph of the introduction.

Other language systems were needed to account for a large number of openings dealing in broad terms with the concept of "contrast". However this had to be broadened considerably to recognize such pairs as comparative denial, denial of the expected, concession, known-new, and hypothetical-real. These all had a surface pattern of being binary and having typical transition markers (yet, but, however, etc.) between the two parts of the structure. A very large number of the articles studied had such a dominant signal in the first few paragraphs, and these important structures demand further detailed analysis.

A few openings could not be classified under either of these two major linguistic systems, and these are discussed under the broad concepts of description of noteworthy or key events, general or re-enacted accounts, description of things, and detailing of known information. While some of these could be viewed as essential to the informational structure of the article, others were questionable in this role, and could perhaps be justified only in terms of colour. By and large, though, the "interest" in the articles was created through the inherent appeal of the search for 
knowledge and understanding explained in the articles rather than through any particular linguistic technique.

So what should we do as teachers of technical writing to help our students create good openings to quite formal scientific texts? First, we need to make sure that they understand principles of the logical connections - not just as essential language constructs, but as vital principles of scientific enquiry, verification and debate. And we also need to help them understand the prevalent need for the many binary pairs of information structure, and again their significance in the real world of science and technology.

We should help students to write openings that reflect the scientific thinking and analysis of their writing, and that therefore help readers to understand the underlying scientific principles involved. Where that is not possible, we need to make students aware of the uses, and potential dangers, of description and anecdotal openings - and especially the importance of making such openings clearly relevant to the thrust of the article.

Other patterns of openings are likely to be found in a more thorough analysis of many more articles than could be examined for this pilot study, and we now need to work on very formal scientific texts, to determine whether the structures outlined here apply in scientific papers - and hopefully to discover other methods too.

\section{REFERENCES}

Grimes, J. (1975) The Thread of Discourse. The Hague: Mouton.

Hoey, M.P. (1983) On the Surface of Discourse. London: George Allen and Unwin.

Jordan, M.P. (1984) Rhetoric of Everyday English Texts. London: George Allen and Unwin. 
Jordan, M.P. (1985a) "Towards an Integrated Theory of Textual Cohesion". Midland Association for Linguistic Study. University of Birmingham, England, New Series 10.

Jordan, M.P. (1985b) "Some Relations of Surprises and Expectation in English". In J. Hall (ed.), The 121th LACUS Forum. Columbia SC: Hornbeam Press.

Jordan, M.P. (1986) "The Language of Evaluation". Technostyle 6(2).

Jordan, M.P. (1989) "Beyond Impressionism: Evaluating Causal Connections." In B.F. Fearing and W.K. Sparrow (eds.) Technical Writing: Theory and Practice. Modern Languages Association.

Jordan, M.P. (1991a) "Openings in Informal Scientific Texts". Technostyle 9(2).

Jordan, M.P. (1991b) "An Integrated Three-Pronged Analysis of a FundRaising Letter". In W.C. Mann and S.A. Thompson (eds.) Discourse Description of a Fund-Raising Letter. Amsterdam: John Benjamins.

Mann, W.C. and S.A. Thomspon (1986) "Relational Propositions in Discourse". Discourse Processes.

Winter, E.O.O. (1974) "Replacement as a Function of Repetition". Ph.D. Thesis, University of London, England (see Dissertation Abstracts International, sec. C, Autumn 1977, 38, 4).

Winter, E.O.O. (1976) "Fundamentals of Information Structure". Hatfield, England: The Hatfield Polytechnic.

Winter, E.O.O. (1982) Towards a Contextual Grammar of English. London: George Allen and Unwin. 